\title{
Definition of Complete Spinal Cord Injury
}

\author{
R. L. Waters, MD, ${ }^{1}$ R. H. Adkins, PhD $^{2}$ J. S. Yakura, MS, PT $^{3}$

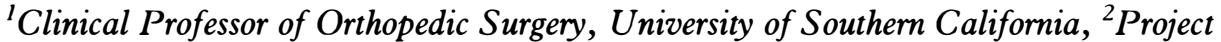 \\ Co-Director, Regional Spinal Cord Injury Care System of Southern California, \\ ${ }^{3}$ Regional Spinal Cord Injury Care System, of Southern California, Rancho Los \\ Amigos Medical Center HB-206, 7601 E, Imperial Highway, Downey, California \\ 90242, USA.
}

\section{Summary}

Prospective serial neurological examinations were performed on 445 consecutive traumatic spinal cord injury (SCI) patients admitted for rehabilitation on an average of $22 \cdot 8 \pm 15 \cdot 6$ days after injury. Patients were categorized by both the ASIA and Sacral Sparing (SS) definitions of complete SCI, in order to compare the definitions in terms of consistency and prognostic ability. Recovery during follow-up was determined by sensory scores for light touch, sharp/dull discrimination, proprioception, and the ASIA Motor Index Score.

Change in complete status was unidirectional using the SS definition and bidirectional using the ASIA definition. Twelve patients with SS complete injuries on initial examination converted to $S S$ incomplete injuries at follow-up. No patients converted from SS incomplete to SS complete injury. Twenty three patients with ASIA complete injuries upon admission converted to ASIA incomplete status and 6 converted from ASIA incomplete status on admission to ASIA complete status at follow-up.

For quadriplegics, the average motor recovery for patients changing complete status according to the ASIA definition was $11 \cdot 7 \pm 10 \cdot 3$, which was significantly less $(p<\cdot 05)$

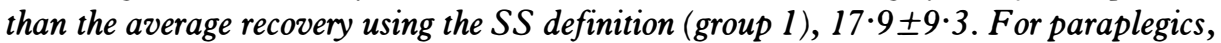
the average motor recovery using the ASIA definition, $8 \cdot 3 \pm 6 \cdot 7$, did not differ significantly from the value using the $S S$ definition, $6 \cdot 8 \pm 4 \cdot 0$.

Key words: Complete spinal cord injury; Sacral Sparing; Zone of Spinal Injury.

The severity of spinal cord trauma is clinically determined by the completeness of the injury based on the neurological examination. Classification of injury completeness assists the clinician in prognosis associated with recovery. ${ }^{1,2}$ Patients with complete injuries persisting after the initial acute injury phase have a less favourable recovery potential than those with incomplete injuries. ${ }^{2,}{ }^{3}$ Two definitions of complete spinal cord injury (SCI) are in common use. According to the

Funded by the National Institute of Disability Rehabilitation Research, Spinal Cord Injury Model Systems Program, Grant No. G008535134. 
Standards for Neurological Classification published by the American Spinal Injury Association (ASIA), ${ }^{4}$ the term complete injury '. . . means no preservation of motor and/or sensory function exists more than 3 segments below the neurological level of injury'. Incomplete injury ' . . . means some preservation of sensory and/or motor function exists more than 3 segments below the neurological level of injury.'

By ASIA definition, the criteria for complete SCI depends on the neurological level of injury (NLI) and the width of the zone of partial preservation (ZPP). The $\mathrm{NLI}$ is defined as 'the most caudal segment that tests as normal, or intact for both sensory and motor functions' on both sides of the body. ${ }^{4}$ If a muscle has at least a grade of 'Fair', it is considered intact when the next most rostral key muscle is tested either 'good' or 'normal'. The ZPP may include up to three consecutive segments caudal to the level of injury having partial motor and/or sensory function. ${ }^{4}$ If sensory and/or motor function ' . . . is found beyond the third segment, the lesion is incomplete and the term ZPP is not used. ${ }^{4}$

The Sacral Sparing (SS) definition of the completeness of injury is simpler than the ASIA definition because it does not depend on the NLI and the ZPP. According to the SS definition, a complete SCI is one in which there is no sensory and/or motor function at the sacral segments and incomplete injury is one in which there is some preservation of sacral motor and/or sensory function. ${ }^{2}$

The study was undertaken to evaluate the ASIA and SS definitions of complete SCI.

\section{Methods}

Examinations of motor and sensory function were performed on 445 consecutive SCVI patients admitted to Rancho Los Amigos Medical Center for rehabilitation between 1985 and 1990. Of this population, 270 were paraplegic injuries and 175 were quadriplegic injuries. There were 397 men and 48 women ranging in age from 16 to 59 years upon admission. The average time from injury to admission was $22 \cdot 8 \pm 15 \cdot 6$ days. The average follow-up was $448 \cdot 2 \pm 469 \cdot 0$ days. The aetiologies of injury are listed on Table I.

The initial examination occurred on admission, and was repeated monthly for 6 months, and then annually, from the patient's date injury. The motor and sensory examinations were performed according to the Standards of the American Spinal Injury Association. ${ }^{4}$ The ASIA Motor Index Score uses standard manual muscle testing on a six grade scale (absent $=0$; trace $=1$, visible or palpable contraction; poor $=2$, active movement through range of motion with gravity eliminated; fair $=3$, active movement through range of motion against gravity; good $=4$, active movement through range of motion against resistance; and normal=5). The key muscles/functions included in the ASIA Motor Index Score are: elbow flexors, wrist extensors, elbow extensors, finger flexors, hand intrinsics, hip flexors, knee extensors, ankle dorsiflexors, long toe extensors and ankle plantar flexors.

The ASIA standards do not directly address detailed sensory assessment, however, the sensory dermatome chart recommended by ASIA was used. Light touch and sharp/dull sensation were tested for each sensory dermatome and graded on a three point scale (absent $=0$; impaired $=1$; and normal $=2$ ). Proprioception was tested at the shoulder, elbow, wrist, thumb, hip, knee, ankle and great toe also using a three point scale. Numerically, a total motor score of 100 , light touch score 
Table I Aetiology of injury

\begin{tabular}{lr}
\hline Aetiology & Frequency \\
\hline Gunshot wound & 174 \\
Motor vehicle accident & 113 \\
Fall & 63 \\
Motor cycle accident & 28 \\
Stab & 11 \\
Auto versus pedestrian & 9 \\
Diving & 8 \\
Assault & 7 \\
Ischaemia & 6 \\
Bicycle & 6 \\
Surfing & 5 \\
Wrestling & 4 \\
Falling object & 3 \\
Football & 3 \\
Skydiving & 2 \\
Sailing & 2 \\
Basketball & 1 \\
Total & \\
\hline
\end{tabular}

of 116, sharp/dull discrimination score of 116 and proprioception score of 32 are possible.

Regarding test reliability, Klose evaluated a similar neurological assessment instrument using three examiners and multiple correlation. ${ }^{3}$ The results of this study indicated remarkably high reliability for both the motor and sensory scales. In this study although one examiner (JY) performed all examinations, inter-rater reliability between the primary examiner and back-up examiners were periodically performed as a quality assurance measure for consistency and as a precautionary measure in the event that a back-up examiner might be required. Overall reliability for motor examinations was $\mathrm{r}=\cdot 9912$, for sharp/dull and light discrimination the reliability figures were $\cdot 9373$ and $\cdot 9343$ respectively.

The presence of sacral motor sparing was determined by the presence of motor function in the external anal sphincter or the toe flexor muscles; however, the grades of these muscles were not included in the ASIA Motor Score. The presence of sacral sensation was determined by the presence of sensation in the perineum at the anal mucocutaneous junction, glans penis or clitoris. If both the sacral motor and sensory sparing were absent, the patient was considered a SS complete injury. The subjects were classified in three main groups: Group 1 included patients who met both the ASIA and Sacral Sparing criteria for complete injury on initial examination and who subsequently converted to incomplete status by both criteria at follow-up; Group 2 included patients who changed status according to the ASIA criteria from complete to incomplete injury or from incomplete to complete injury but remained complete according to the Sacral Sparing definition; Group 3 consisted of patients who met both the ASIA and SS criteria for complete injury on admission and throughout follow-up.

Patients were classified according to their initial NLI. The width of the ZPP for motor, sharp/dull discrimination and light touch sensation were measured for each case. The ASIA Motor Index increase between initial and final exams was used as 
an indicator of motor recovery. The increase between initial and final light touch, sharp/dull discrimination and.proprioception scores was used to measure sensory recovery.

Sample descriptive statistics, frequency distributions and cross tabulations were conducted to summarize attributes of the sample. Analysis of variance with appropriate post-hoc tests were employed to assess differences between groups with regard to the amount of recovery.

\section{Results}

There were 216 subjects without sacral sparing on initial examination who were SS complete. Of these, there were 209 subjects lacking sensory or motor function more than 3 segments below the NLI who were ASIA complete.

Change in complete status was in one direction using the SS definition and in two directions using the ASIA definition. Twelve patients with SS complete injuries on initial examination converted to SS incomplete injuries at follow-up. No patients converted from SS incomplete to SS complete injury. Twenty three patients with ASIA complete injuries converted to ASIA incomplete status and 6 converted from ASIA incomplete status on admission to ASIA complete status at follow-up. Thus, 12 (3\%) of all 445 patients unidirectionally changed completeness status according to the SS definition and $29(7 \%)$ bidirectionally changed status using the ASIA definition.

All 12 patients converting from SS complete to SS incomplete status also converted from complete to incomplete status using the ASIA definition. These 12 patients comprised Group 1.

Eleven patients converted from ASIA complete to ASIA incomplete status at follow-up but had no sacral sparing on admission and at follow-up remaining SS complete. Six patients coverted from ASIA incomplete to ASIA complete injuries according to the ASIA criteria but had no sacral sparing and remained SS complete. This was due to the recovery of motor and/or sensory function causing the NLI to descend and narrow the ZPP to less than 4 segments. One additional patient without sacral sparing was ASIA incomplete on admission and follow-up since light touch sensation on admission spanned four neurological segments below the level of injury. On examination 2 months after injury, the neurological level of injury had descended one level so that sensation spanned only three levels below the level of injury meeting the ASIA criteria for a complete injury. However, on examination 6 months after injury he had one level further caudal sensory recovery so that sensation was present four segments below the level of injury meeting the criteria for an ASIA incomplete injury. These 18 patients were assigned to Group 2.

The majority of patients, 186 cases, were in group 3 and met both the SS and ASIA criteria for complete SCI on initial examination and during follow-up.

\section{Group 1}

The time interval for conversion from complete to incomplete status, total days of follow-up and ASIA motor index recovery for Group 1 are listed on Table II. Seven were quadriplegic and 5 were paraplegic of injuries. All were 'late conversions' 
Table II Group $1^{+}$

\begin{tabular}{|c|c|c|c|c|}
\hline Patients & $\begin{array}{l}\text { Conversion* } \\
\text { (ASIA and SS) }\end{array}$ & $\begin{array}{l}\text { Conversion interval } \\
\text { (days) }\end{array}$ & $\begin{array}{l}\text { Length FU } \\
\text { (days) }\end{array}$ & $\begin{array}{c}\text { ASIA } \\
\text { Motor score } \\
\text { recovery }\end{array}$ \\
\hline \multicolumn{5}{|c|}{ Quadriplegia } \\
\hline 1 & $\mathrm{C}$ to $\mathrm{I}$ & $31-58$ & 140 & 37 \\
\hline 2 & $\mathrm{C}$ to $\mathrm{I}$ & $61-88$ & 369 & 9 \\
\hline 3 & $\mathrm{C}$ to $\mathrm{I}$ & $120-210$ & 381 & 20 \\
\hline 4 & $\mathrm{C}$ to $\mathrm{I}$ & $59-89$ & 120 & 16 \\
\hline 5 & $\mathrm{C}$ to I & $332-916$ & 916 & 16 \\
\hline 6 & $\mathrm{C}$ to $\mathrm{I}$ & $60-88$ & 1029 & 17 \\
\hline 7 & $\mathrm{C}$ to $\mathrm{I}$ & $120-149$ & 341 & 10 \\
\hline \multicolumn{5}{|c|}{ Paraplegia } \\
\hline 8 & $\mathrm{C}$ to $\mathrm{I}$ & $161-385$ & 385 & 0 \\
\hline 9 & $\mathrm{C}$ to $\mathrm{I}$ & $62-115$ & 388 & 9 \\
\hline 10 & $\mathrm{C}$ to $\mathrm{I}$ & $90-409$ & 409 & 14 \\
\hline 11 & $\mathrm{C}$ to $\mathrm{I}$ & $372-454$ & 454 & 7 \\
\hline 12 & $\mathrm{C}$ to $\mathrm{I}$ & $160-391$ & 901 & 4 \\
\hline
\end{tabular}

* Conversion of Complete Status by both ASIA and Sacral Sparing Criteria.

+ Group 1 consisted of patients who underwent conversion from complete SCI to incomplete SCI during follow up by both the SS and ASIA criteria.

$\mathrm{C}=$ Complete SCI; I = Incomplete SCI; FU = Follow-up.

Table III Number of patients in subgroups

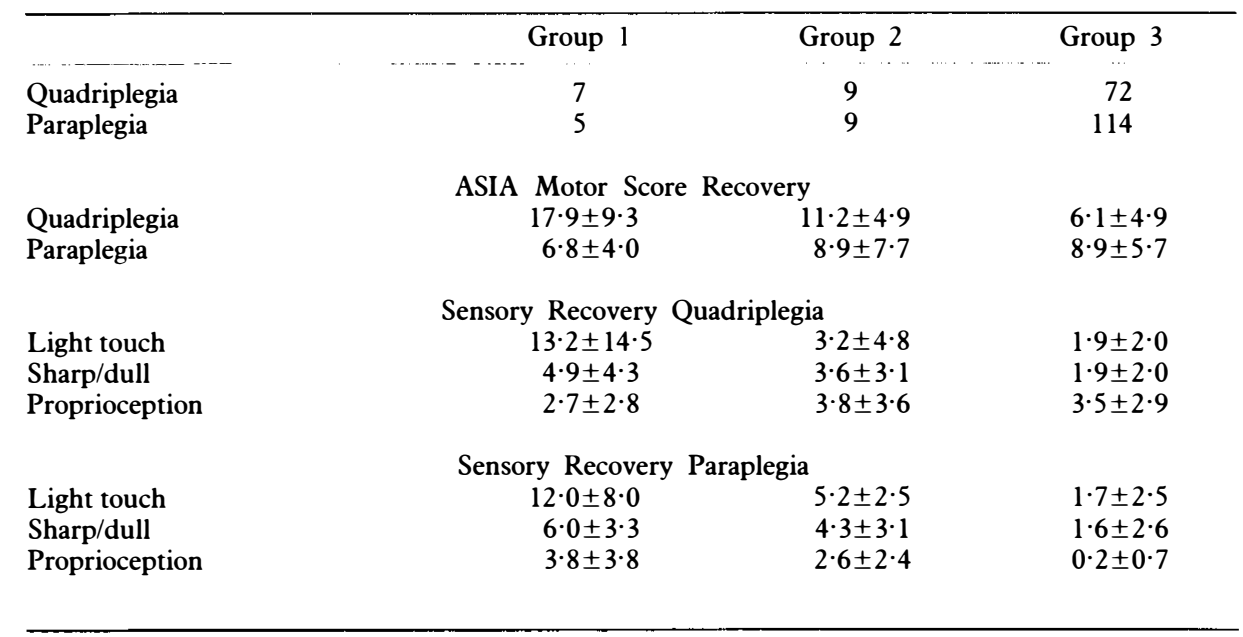

occurring more than 30 days after injury. The conversion to incomplete status occurred within the first 7 months post injury in 6 of the 7 quadriplegic injuries, and within 15 months post injury for the paraplegic injuries.

The average motor recovery for the quadriplegics in Group 1, 17.9 $\pm 9 \cdot 3$, was significantly higher than the value for quadriplegic Group 2 patients, $11 \cdot 2 \pm 4 \cdot 9$ $(\mathrm{p}<\cdot 05)$ and for quadriplegic Group 3 controls, 6.1 $\pm 4 \cdot 9(\mathrm{p}<\cdot 0001)$. For paraplegics, there were no significant differences in motor recovery between Group $1,6 \cdot 8 \pm 4 \cdot 0$, group $2,8 \cdot 9 \pm 7 \cdot 7$ or Group $3,8 \cdot 9 \pm 5 \cdot 7$ (Table III). 
The mean light touch, sharp/dull and proprioception recoveries for Group 1 patients are listed in Table III. For light touch, recovery in Group 1 was significantly greater than that in Group $2(\mathrm{p}<\cdot 0001)$ and Group $3(\mathrm{p}<\cdot 0001)$. For sharp/dull discrimination, recovery in Group 1 was significantly greater than that in group 3; however, the difference between Group 1 and Group 2 was not significant. Similarly, for recovery of proprioception, Group 1 gains were significantly greater than those of Group $3,(p<\cdot 004)$, but the difference between Group 1 and 2 was not significant, in fact the means were identical. In addition, the amount of light touch recovery in Group 1 was significantly $(\mathrm{p}<\cdot 05)$ greater than recovery of sharp/dull discrimination for both quadriplegics and paraplegics.

\section{Group 2}

The initial and final NLI and maximum length of the motor ZPP, sharp/dull discrimination ZPP and light touch ZPP during follow up are listed for Group 2 on Table IV.

Table IV Group 2

\begin{tabular}{|c|c|c|c|c|c|}
\hline Patient & Initial NLI* & Final NLI** & Motor ZPP*** & $\begin{array}{c}\text { Sharp/dull }{ }^{* * * *} \\
\text { ZPP }\end{array}$ & $\begin{array}{l}\text { Light touch } \\
\mathrm{ZPP}^{* * *}\end{array}$ \\
\hline \multicolumn{6}{|c|}{ Quadriplegia } \\
\hline \multicolumn{6}{|c|}{ ASIA complete to ASIA incomplete } \\
\hline 1 & $\mathrm{C} 3$ & $\mathrm{C} 4$ & 3 & 2 & 4 \\
\hline 2 & $\mathrm{C} 3$ & $\mathrm{C} 4$ & 3 & 2 & 7 \\
\hline 3 & $\mathrm{C} 4$ & $\mathrm{C} 5$ & 3 & 3 & 4 \\
\hline 4 & C6 & $\mathrm{C} 7$ & 1 & 1 & 4 \\
\hline \multicolumn{6}{|c|}{ ASIA incomplete to ASIA complete } \\
\hline 5 & $\mathrm{C} 5$ & $\mathrm{C} 8$ & 4 & 4 & 5 \\
\hline 6 & $\mathrm{C} 5$ & $\mathrm{C} 5$ & 2 & 1 & 4 \\
\hline 7 & $\mathrm{C} 5$ & $\mathrm{C} 8$ & 4 & 3 & 4 \\
\hline 8 & $\mathrm{C} 3$ & $\mathrm{C} 4$ & 2 & 2 & 4 \\
\hline 9 & $\mathrm{C} 4$ & C6 & 4 & 3 & 3 \\
\hline \multicolumn{6}{|c|}{ Paraplegia } \\
\hline \multicolumn{6}{|c|}{ ASIA complete to ASIA incomplete } \\
\hline 10 & $\mathrm{~T} 10$ & $\mathrm{~T} 10$ & 0 & 1 & 4 \\
\hline 11 & $\mathrm{~T} 10$ & T12 & 4 & 4 & 4 \\
\hline 12 & T11 & T11 & 4 & 3 & 6 \\
\hline 13 & $\mathrm{~T} 12$ & $\mathrm{~T} 12$ & 4 & 3 & 3 \\
\hline 14 & $\mathrm{~T} 12$ & L2 & 4 & 3 & 3 \\
\hline 15 & $\mathrm{~T} 12$ & $\mathrm{~T} 12$ & 3 & 2 & 4 \\
\hline 16 & $\mathrm{~T} 12$ & L1 & 3 & 3 & 4 \\
\hline \multicolumn{6}{|c|}{ ASIA incomplete to ASIA complete } \\
\hline 17 & $\mathrm{~T} 12$ & L2 & 3 & 4 & 5 \\
\hline \multicolumn{6}{|c|}{ ASIA incomplete to ASIA complete to ASIA incomplete } \\
\hline 18 & $\mathrm{~T} 12$ & L1 & 3 & 3 & 4 \\
\hline
\end{tabular}

+ Group 2: Patients remaining SS complete injuries throughout follow-up but converting completeness status according to ASIA criteria.

*Initial neurological level of injury.

**Final neurological level of injury.

***Number of levels within zone of partial preservation.

ZPP Zone of partial preservation. 
Four quadriplegic and seven paraplegic injuries initially classified as ASIA complete converted to ASIA incomplete injuries during follow-up by acquiring more than 3 segments of motor or sensory function beyond the NLI. In 9 of the 11 patients, the conversion occurred within the first year months after injury.

All 4 quadriplegic injuries became incomplete by extending the number of segments having light touch to 4 or more segments beyond the NLI, but the motor and sharp/dull discrimination ZPP was within 3 segments (Table V). The initial NLI of all 7 paraplegics was at or below T10. Among these paraplegics, 5 had light touch, 1 had sharp/dull discrimination, and 4 had motor function at four or more segments below the NLI (Table V).

Five patients who were quadriplegic and one who was paraplegic from injury initially had more than 3 segments of partial sensory and/or motor function beyond the NLI, and upon follow up had 3 or less segments beyond the NLI. These patients met the criteria for conversion from incomplete to complete status by the ASIA criteria. Despite conversion from incomplete to complete status, all 6 patients gained at least some motor function (Table V). Conversion in 5 of the 6 patients occurred within the first year. In one patient conversion occurred after 3 years.

The average motor recovery in the nine Group 2 quadriplegics, $11 \cdot 2 \pm 4.9$ was

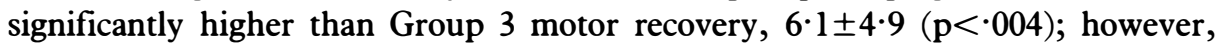

Table V Group 2

\begin{tabular}{|c|c|c|c|c|}
\hline Patients & $\begin{array}{l}\text { Conversion* } \\
\text { (ASIA only) }\end{array}$ & $\begin{array}{c}\text { Conversion interval } \\
\text { (days) }\end{array}$ & $\begin{array}{c}\text { Total FU } \\
\quad \text { (days) }\end{array}$ & $\begin{array}{l}\text { ASIA Motor } \\
\text { recovery }\end{array}$ \\
\hline \multicolumn{5}{|c|}{ Quadriplegia } \\
\hline 1 & $\mathrm{C}$ to $\mathrm{I}$ & $88-120$ & 1114 & 6 \\
\hline 3 & $\mathrm{C}$ to $\mathrm{I}$ & $31-59$ & 677 & 12 \\
\hline 5 & $\mathrm{C}$ to $\mathrm{I}$ & $90-122$ & 1185 & 12 \\
\hline 9 & $\mathrm{C}$ to $\mathrm{I}$ & $122-271$ & 271 & 10 \\
\hline 6 & I to $\mathrm{C}$ & $89-117$ & 837 & 6 \\
\hline 7 & I to $\mathrm{C}$ & $122-148$ & 179 & 5 \\
\hline 8 & I to $\mathrm{C}$ & $1045-1548$ & 1548 & 18 \\
\hline 2 & I to $\mathrm{C}$ & $31-61$ & 363 & 18 \\
\hline 4 & I to $\mathrm{C}$ & $30-60$ & 1185 & 14 \\
\hline \multicolumn{5}{|c|}{ Paraplegia } \\
\hline 10 & $\mathrm{C}$ to $\mathrm{I}$ & $89-129$ & 712 & 0 \\
\hline 11 & $\mathrm{C}$ to $\mathrm{I}$ & $90-117$ & 357 & 3 \\
\hline 12 & $\mathrm{C}$ to $\mathrm{I}$ & $90-131$ & 186 & 2 \\
\hline 13 & $\mathrm{C}$ to $\mathrm{I}$ & $365-1068$ & 1068 & 5 \\
\hline 15 & $\mathrm{C}$ to $\mathrm{I}$ & $59-128$ & 730 & 22 \\
\hline 16 & $\mathrm{C}$ to $\mathrm{I}$ & $415-839$ & 839 & 14 \\
\hline 17 & $\mathrm{C}$ to $\mathrm{I}$ & $184-366$ & 366 & 16 \\
\hline 14 & I to $\mathrm{C}$ & $28-62$ & 1037 & 14 \\
\hline 18 & $I$ to $C$ to $I^{* * *}$ & NA & 171 & 4 \\
\hline
\end{tabular}

+Group 2: Patients remaining SS complete injuries throughout follow-up but converting completeness status according to ASIA criteria.

*Conversion of Complete Status by ASIA criteria (no sacral sparing).

**See text for discussion.

$\mathrm{C}=$ Complete SCI; I = Incomplete SCI; FU=Follow-up. 
motor recovery in Group 2 and Group 3 paraplegics was not significantly different. Furthermore, values for light touch, sharp/dull discrimination and proprioception recovery were significantly higher $(\mathrm{p}<\cdot 005)$ for Group 2 than for Group 3 (Table III).

\section{Group 3}

The majority of patients ( 72 quadriplegics and 114 paraplegics) having a diagnosis of a complete injury by both the ASIA and SS definitions remained complete throughtout follow-up. The average motor and sensory recovery scores for Group 3 are listed in Table III.

\section{Late conversion to incomplete status}

All patients converting from complete to incomplete status using either the ASIA or SS definition were 'late conversions' occurring more than 30 days after injury. The SS complete patients who underwent late conversion to incomplete status consist of all Group 1 patients. There were 23 patients undergoing late conversion to incomplete status using the ASIA definition consisting of all Group 1 patients and eleven patients in Group 2 (Table IV and V).

For quadriplegics, the average motor recovery for late ASIA conversion was $11 \cdot 7 \pm 10 \cdot 3$ which was significantly less $(p<\cdot 05)$ than the value using the SS

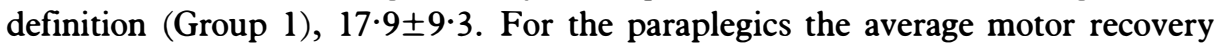
using the ASIA definition, $8 \cdot 3 \pm 6 \cdot 7$, did not differ significantly from the value using the SS definition, $6 \cdot 8 \pm 4 \cdot 0$ or for Group 3 controls, $8 \cdot 9 \pm 5 \cdot 7$.

\section{Discussion}

Immediately after SCI, a state of spinal shock develops which may result in areflexia or hyporeflexia for varying time periods. A patient at this time may have no motor or sensory function below the level of the lesion and still regain excellent neurological function limiting the validity and usefulness of classifying patients for prognostic purposes as complete injuries in the acute phase. However, as the length of time increases after injury, persistence of a complete injury progressively diminishes the chance for motor and sensory recovery. Patients in this series were examined subacutely at the beginning of rehabilitation and those having a diagnosis of a complete injury by either the ASIA or SS definition had a relatively small amount of motor and sensory recovery during follow up in comparison to the total deficit.

For quadriplegic patients undergoing late conversion from complete to incomplete status, the SS definition reflected motor recovery in the extremities more favourably than the ASIA definition. The stability of the SS definition was also greater than the ASIA definition. Change was unidirectional (from complete to incomplete) in $3 \%$ of all cases using the SS definition. Change was bidirectional using the ASIA definition and 7\% of patients changed status during follow-up.

Eleven patients within Group 2 who were initially complete according to both the ASIA and Sacral Sparing definitions gained partial sensory or motor recovery at final follow-up that extended 4 or more segments beyond the level of injury. These 
patients met the criteria for conversion from complete to incomplete injury according to the ASIA definition but not the SS definition. Although the amount of motor recovery was slightly greater for the four Group 2 quadriplegics than the Group 3 quadriplegic controls, the difference was small (10 vs 8.9). From a clinical perspective, the conversion to incomplete status using the ASIA definition represented a 'false positive' change.

Six patients in Group 2 lacking sacral sparing were incomplete on initial evaluation since there were more than 3 segments with partial motor or sensory function beyond the NLI and during follow-up converted to complete status according to the ASIA definition. This was due to the recovery of motor and/or sensory function causing the NLI to descend and narrow the ZPP to less than 4 segments. Therefore, by ASIA definition these patients converted to complete SCI status. Motor recovery was not statistically different than in Group 3 patients that remained complete. From the clinical perspective, the conversion in these patients from incomplete to complete status using the ASIA definition conveyed 'false negative' neurological deterioration.

We conclude that the SS definition of complete injury is a simpler and more stable definition of complete SCI than the ASIA definition.

\section{References}

1. Holdsworth P. Fractures, dislocations and fracture dislocations of the spine. J Bone Joint Surg 1970: 52A:1534-1551.

2. Stauffer S. Diagnosis and prognosis of acute cervical spinal cord injury. Clin Ortho Rel Res 1975: 112:9-15.

3. Klose KJ, Green BA, Smith RS, Adkins RH, MacDonald AM. University of Miami Neuro-Spinal Index (UMNI): A quantitative method for determining spinal cord function. Paraplegia 1980: 18:331-336.

4. American Spinal Injury Association: Standards for Neurological Classification of Spinal Injury Patients. Revised April 1990. 